\title{
Evolution of Light-Induced Vapor Generation at a Liquid-Immersed Metallic Nanoparticle
}

\author{
Zheyu Fang, ${ }^{,}, \dagger, \perp$ Yu-Rong Zhen, ${ }^{\ddagger}$ Oara Neumann, ${ }^{\dagger, \ddagger}$ Albert Polman, ${ }^{\S}$ F. Javier García de Abajo,"
} Peter Nordlander, ${ }^{*,+, \neq}$ and Naomi J. Halas ${ }^{*, t, *}$

\author{
${ }^{\dagger}$ Department of Electrical and Computer Engineering and ${ }^{\ddagger}$ Department of Physics and Astronomy and Laboratory for \\ Nanophotonics, Rice University, 6100 Main Street, Houston, Texas 77005, United States \\ ${ }^{\S}$ Center for Nanophotonics, FOM Institute AMOLF, Science Park 104, 1098 XG Amsterdam, The Netherlands \\ "IQFR-CSIC, Serrano 119, 28006 Madrid, Spain \\ ${ }^{\perp}$ School of Physics, State Key Lab for Mesoscopic Physics, Peking University, Beijing 100871, China
}

\section{Supporting Information}

\begin{abstract}
When an Au nanoparticle in a liquid medium is illuminated with resonant light of sufficient intensity, a nanometer scale envelope of vapor-a "nanobubble" - surrounding the particle, is formed. This is the nanoscale onset of the wellknown process of liquid boiling, occurring at a single nanoparticle nucleation site, resulting from the photothermal response of the nanoparticle. Here we examine bubble formation at an individual metallic nanoparticle in detail. Incipient nanobubble formation is observed by monitoring the plasmon resonance shift of an individual, illuminated $\mathrm{Au}$ nanoparticle, when its local environment changes from liquid to vapor. The temperature on the nanoparticle surface is monitored during this process, where a dramatic temperature jump is observed as the nanoscale vapor

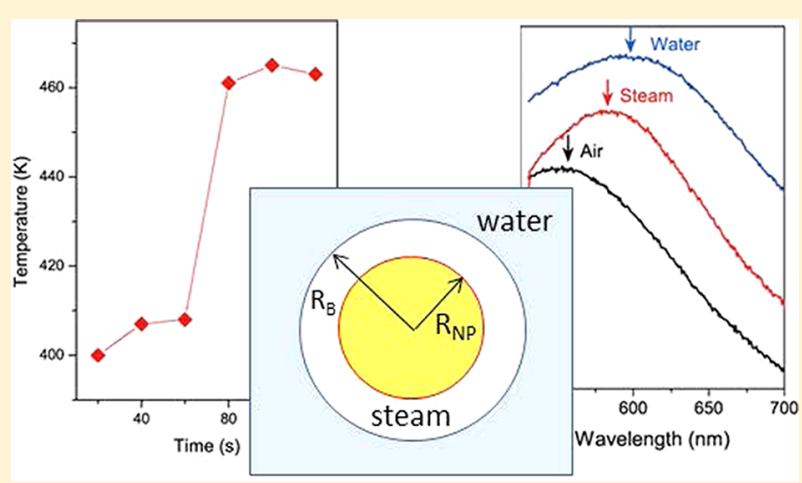
layer thermally decouples the nanoparticle from the surrounding liquid. By increasing the intensity of the incident light or decreasing the interparticle separation, we observe the formation of micrometer-sized bubbles resulting from the coalescence of nanoparticle-"bound" vapor envelopes. These studies provide the first direct and quantitative analysis of the evolution of lightinduced steam generation by nanoparticles from the nanoscale to the macroscale, a process that is of fundamental interest for a growing number of applications.
\end{abstract}

KEYWORDS: Plasmonic heating, Au nanoparticle, LSPR, nanobubble, microbubble

$\mathrm{R}$ ecently it was demonstrated that metallic nanoparticles, suspended in liquids and illuminated by focused sunlight, can produce steam with remarkably high efficiency. ${ }^{1}$ More than $80 \%$ of the power absorbed by the nanoparticles resulted in vaporization, while less than $20 \%$ of the absorbed power went into heating the liquid. ${ }^{1}$ This result, occurring at a very low light intensity threshold $\left(\sim 10^{6} \mathrm{~W} / \mathrm{m}^{2}\right)$, has stimulated significant interest in the fundamental aspects of this process as well as its possible technological applications. ${ }^{2}$ To develop and optimize this effect for applications, which range from medical and waste sterilization to water purification and distillation, it is crucial to develop a fundamental microscopic understanding of the vaporization process. Although no microscopic theory was proposed to explain the measured efficiencies, the authors noted that the results were consistent with a very simple model where a vapor envelope nucleates around each individual illuminated nanoparticle. The nanoparticle/vapor bubble complex expands under further illumination, eventually achieving buoyancy, moving toward the liquid/air interface and releasing the vapor, after which the nanoparticle reverts back into the solution and, as long as it is illuminated, repeats the process. Clearly an essential step in this process is the initial formation of a vapor layer around the illuminated nanoparticle.

Noble metal nanoparticles, when illuminated at a wavelength corresponding to their plasmon resonance, can serve as highly efficient localized heat sources at the nanometer-length scale. $^{3-18}$ This is a unique and well-established property that has enabled numerous applications across a range of fields including energy, ${ }^{19-21}$ chemical catalysis, ${ }^{4,8}$ protein imaging, ${ }^{10,12}$ and biomedicine. ${ }^{14,17}$ Several studies have also investigated bubble and vapor formation in ensembles of metallic nanoparticles under continuous-wave (CW) and pulsed laser excitation. ${ }^{3-5,13,16,22,23}$ Despite these studies, the precise properties of the liquid-vapor phase transition at the nanoparticle surface, such as temperature, internal pressure, and size of the vapor envelope - the "nanobubble"-formed upon

Received: January 25, 2013

Revised: $\quad$ March 11, 2013

Published: March 21, 2013 
resonant laser excitation have not been investigated. Quantifying these properties at the single nanoparticle level is crucial for understanding the vapor generation process.

In this Letter, we investigate plasmon-induced vapor generation from an individual nanoparticle deposited on a substrate. We combine dark-field scattering measurements of localized surface plasmon resonance (LSPR) shifts with surfaceenhanced Raman scattering (SERS)-based temperature measurements on individual, illuminated Au nanoparticles. These nano-optic probes were performed during controlled resonant laser excitation at incident intensities both below and above the threshold required to induce local vapor formation. This combination of local optical measurements at an individual nanoparticle allows us to determine several key microscopic parameters of this system, such as the nanobubble radius around the nanoparticle surface, the internal nanobubble pressure, the nanoparticle surface temperature, and their dependence on incident light intensity. For dense nanoparticle coverages and with further increases in incident light intensity, we observe the formation of micrometer sized bubbles due to the coalescence of nanobubbles centered on adjacent nanoparticles. We quantify the energetics of macroscopic bubble formation based on the incident laser power and the areal density of nanoparticle nucleation sites. This series of experiments provides a detailed picture of the light-induced steam formation process by resonant excitation of metallic nanoparticles which will be useful for the development and optimization of applications of this unique phenomenon.

Gold nanoparticles were chemically synthesized and then immobilized with an ultrathin layer of poly(4-vinyl pyridine)(PVP) onto a transparent dielectric substrate at the bottom of a microchamber (Figure 1a). These nanoparticles were dispersed onto the substrate surface at a very dilute areal coverage, nominally 1 nanoparticle/100 $\mu \mathrm{m}^{2}$ (Figure $1 \mathrm{~b}$ shows a SEM image of a sample of deposited nanoparticles at significantly higher areal density, for visualization purposes; more details in the Methods section). The microchamber was designed to be optically probed by dark field microscopy (for LSPR spectroscopy) during simultaneous illumination by a CW "heating" laser beam. The CW resonant heating laser also served as the Raman pump laser for SERS studies of the heating of the nanoparticle surface. The microchamber could be evacuated for studies in ambient air or filled with Deionized (DI) water.

Dark-field optical microspectroscopy ${ }^{24,25}$ was used to detect changes in the individual nanoparticle LSPR scattering spectrum upon resonant laser illumination (Figure 1c). The measured LSPR spectrum in air was found to peak at $563 \mathrm{~nm}$. When the microchamber was filled with DI water with a temperature of $20^{\circ} \mathrm{C}$, the LSPR was observed to redshift to $598 \mathrm{~nm}$. The redshift of $35 \mathrm{~nm}$ is in good agreement with the redshift obtained from a Mie calculation for the same particle embedded in air compared with that in water at $20{ }^{\circ} \mathrm{C}(\varepsilon=$ 1.778). During laser illumination while the nanoparticle was surrounded by water, however, the LSPR was observed to blueshift to $581 \mathrm{~nm}$. The observed shift implies a decreased refractive index of the medium directly surrounding the nanoparticle, which we interpret as resulting from a water vapor envelope surrounding the nanoparticle.

The incident light intensity threshold for the blueshift of the LSPR is approximately $2.5 \times 10^{10} \mathrm{~W} / \mathrm{m}^{2}$, which is much larger than the light intensities used in the nanoparticle-enabled solar steam generation experiment. A likely reason for this difference is that the presence of the substrate forces a nonspherical shape
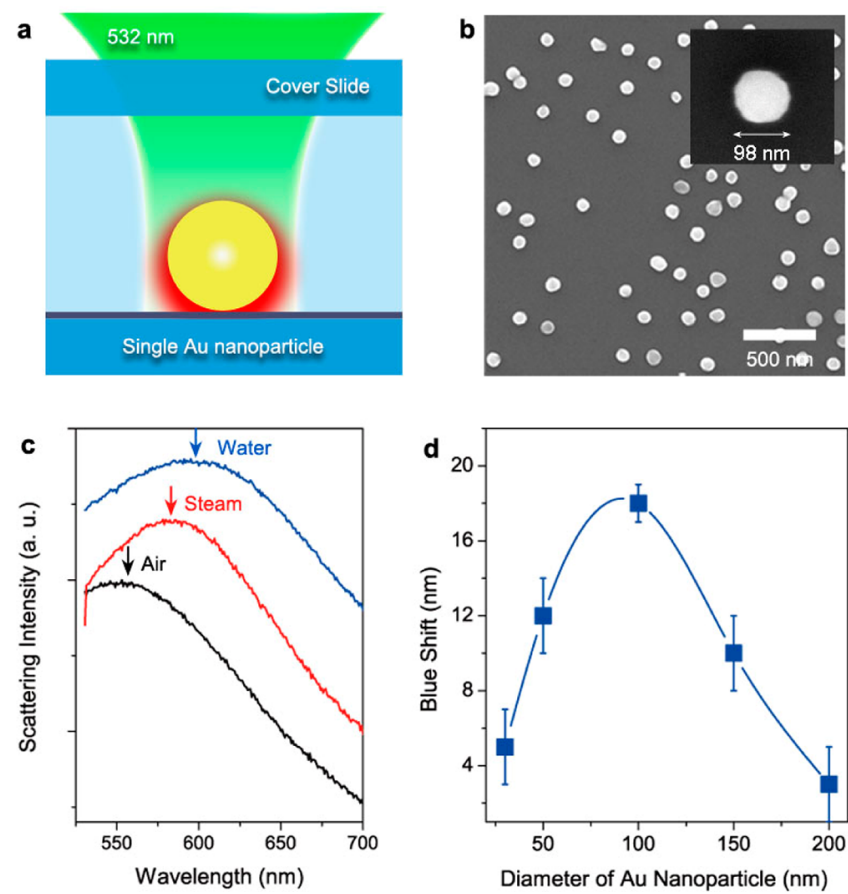

Figure 1. Light-induced generation of a nanobubble at a single nanoparticle surface. (a) Schematic showing illumination and plasmonic heating of an individual Au nanoparticle on the transparent glass substrate floor of our fluidic microchamber. (b) Scanning electron microscope (SEM) images of a sample with a dense coverage of Au nanoparticles randomly distributed on the glass substrate and a typical nanoparticle (inset). (c) Scattering intensities of a single 100 $\mathrm{nm}$ diameter nanoparticle in air (black), water (blue), and an envelope of water vapor produced by laser illumination (red). The blueshift corresponds to formation of a finite vapor envelope of nanometer scale thickness around the nanoparticle. Spectra are shifted vertically for clarity. The red curve corresponds to a vapor envelope thickness of 6.5 $\mathrm{nm}$. (d) Dependence of nanobubble-induced LSPR blueshift on Au nanoparticle diameter, with a maximum shift observed for a nanoparticle diameter of $100 \mathrm{~nm}$. The error bars represent the nanoparticle-to-nanoparticle variation in the LSPR blueshift observed for a set of several $(<10)$ nanoparticles. The incident laser power at $532 \mathrm{~nm}$ was $25 \mathrm{~mW}$ focused to a $1 \mu \mathrm{m}$ diameter spot.

for the nascent vapor bubble. The increased curvature of the vapor bubble near the nanoparticle/substrate interface should result in substantially increased surface tension, making bubble formation in this geometry far more difficult than for an isolated nanoparticle suspended in solution. Other effects that could be present for nanoparticles suspended in solution, such as multiple scattering and collective field enhancement effects, ${ }^{26}$ may also reduce the energy threshold for an isotropic geometry relative to a substrate-bound structure.

When illuminated by a CW laser, an Au nanoparticle is heated due to nonradiative plasmon decay. For low incident laser power, heat from the nanoparticle is transferred into the surrounding solution, resulting in a steady-state thermal gradient. $^{6}$ At higher incident powers $(25 \mathrm{~mW}$ and above), a thin layer of surrounding water vaporizes and forms a nanobubble which provides a thermal barrier, reducing subsequent heat transfer into the surrounding solution. The size of the nanobubble is determined from a simple energy balance: the bubble expands until the power dissipated into the liquid becomes equal to the power absorbed by the nanoparticle from the incident laser light. The observed 

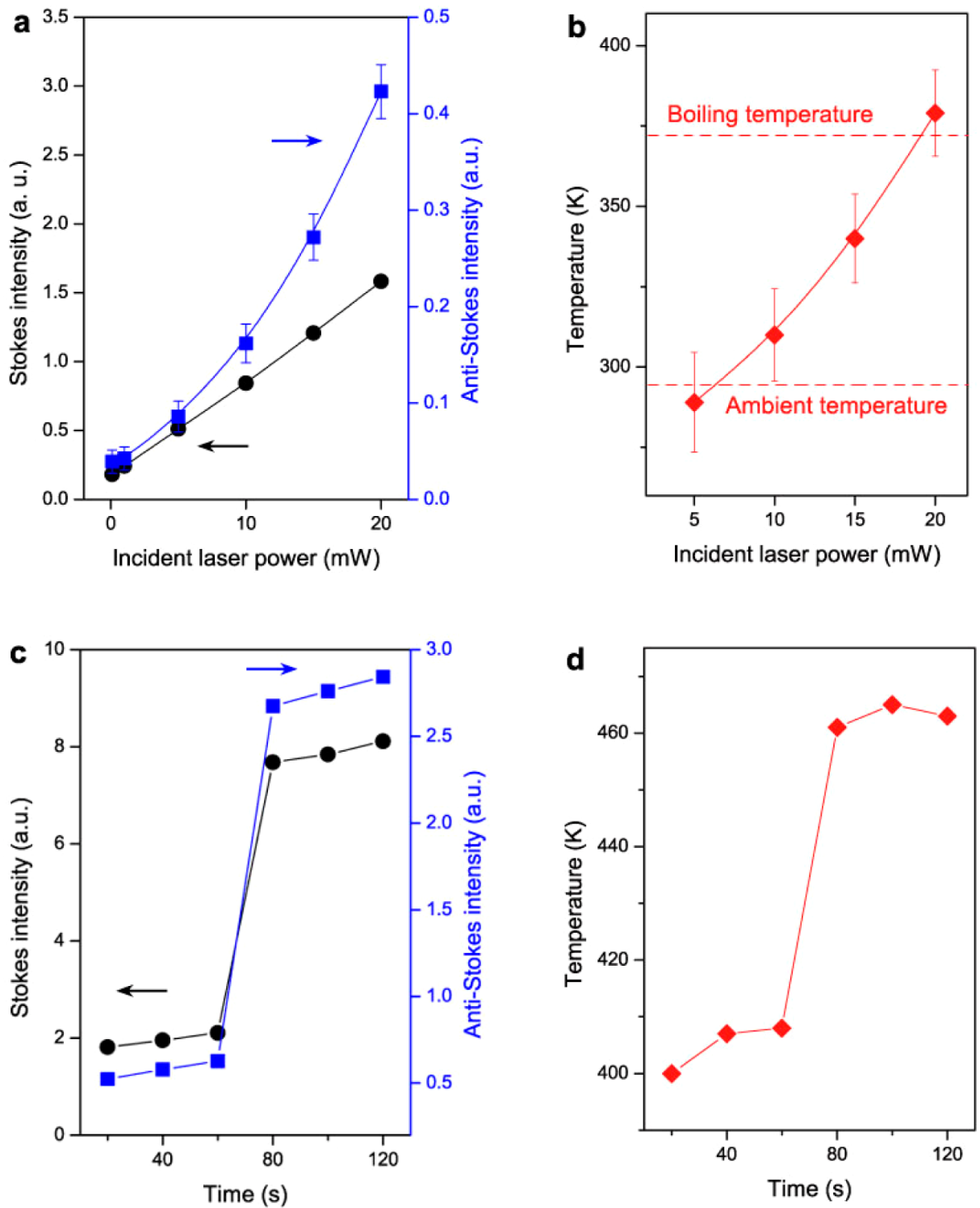

Figure 2. Temperature at the Au nanoparticle surface during the steam generation process. (a) Power dependence of the Au-S Stokes (black dots) and anti-Stokes (blue squares) mode amplitudes at $1390 \mathrm{~cm}^{-1}$ for a pMA-coated single Au NP (100 nm diameter) as a function of incident laser power. (b) Temperature obtained from the mode amplitudes in a and eq 1 , as a function of incident pump laser power. Error bars in panels a and $b$ indicate the uncertainty in the signal due to the low anti-Stokes signal levels in this range of incident laser intensities. (c) Time dependence of Stokes (black dots) and anti-Stokes (blue squares) signals with $25 \mathrm{~mW}$ laser excitation. A large intensity jump occurs after $60 \mathrm{~s}$ of laser excitation. (d) Temperature jump obtained from the mode amplitudes shown in $\mathrm{c}$ and eq 1 occurring after $60 \mathrm{~s}$ of $25 \mathrm{~mW}$ laser excitation. The $532 \mathrm{~nm}$ light was focused on a $1 \mu \mathrm{m}$ diameter spot.

blueshift of the LSPR is far too large to be consistent with merely heating the surrounding liquid: for a gold nanoparticle of an equivalent size immersed in $100{ }^{\circ} \mathrm{C}$ water ambient, only a $3 \mathrm{~nm}$ blueshift would be observed (Figure S1, Supporting Information).

The rate of plasmon-induced heat generation in a nanoparticle is proportional to its optical absorption cross section multiplied by the incident optical intensity. ${ }^{3,4}$ Since the absorption efficiency (absorption cross-section divided by the nanoparticle cross-section $\pi R_{\mathrm{NP}}^{2}$ ) varies with nanoparticle size, the dependence of the blueshift on nanoparticle size was also investigated (Figure 1d). A maximum blueshift of $18 \mathrm{~nm}$ was detected for $\mathrm{Au}$ nanoparticles of $100 \mathrm{~nm}$, decreasing quite strongly for both smaller and larger nanoparticles. This trend clearly shows an optimal nanoparticle size for steam generation at $532 \mathrm{~nm}$ (see Figure S2, Supporting Information). For each illuminated particle, the light-induced heating power is a product of the incident light intensity by the absorption crosssection. For the optimal nanoparticle size of nominally $100 \mathrm{~nm}$ diameter, the laser induced heat generation in each particle is $\sim 5 \times 10^{-4} \mathrm{~W}$. The reason for the reduction in blueshift for large nanoparticles is phase retardation with increasing size. The error bars reflect variations in the resonance blueshift measured for different particles with the same nominal size, which we attribute to the size and shape variation of chemically synthesized $\mathrm{Au}$ nanoparticles in each size range. This variation suggests a sensitivity of the steam nucleation threshold to details of nanoparticle morphology.

To directly measure the temperature at the nanoparticle surface during the bubble formation, we performed SERS on individual $100 \mathrm{~nm}$ diameter $\mathrm{Au}$ nanoparticles. The nanoparticles were functionalized with a monolayer of para- 
mercaptoaniline (pMA, $0.3 \mathrm{~nm}$ thick), shown previously to yield a robust SERS signal from individual nanoparticles under resonant laser pumping. ${ }^{11}$ The SERS measurements were performed in the water-filled microchamber.

Optical vibrational pumping was observed, showing an evolution of the Raman response of the system as a function of incident laser power (Figure 2) (the Stokes and anti-Stokes Raman spectra are shown in Figure S3). While several spectral peaks were observed in the Stokes spectrum, our analysis focused on the $390 \mathrm{~cm}^{-1}$ mode, which corresponds to the $\mathrm{Au}-$ $S$ stretching mode associated directly with the chemical bond between the adsorbate molecules and the $\mathrm{Au}$ nanoparticle surface. A quadratic dependence of the anti-Stokes intensity on incident power is observed, as expected in the optical pumping regime (Figure $2 \mathrm{~b}$ ). ${ }^{27} \mathrm{~A}$ temperature was extracted from the ratio of the Stokes and anti-Stokes intensities for this vibrational mode using ${ }^{11,15,18}$

$$
\frac{I_{\mathrm{AS}}}{I_{\mathrm{S}}}=A\left(\frac{E_{0}+E_{\mathrm{ph}}}{E_{0}-E_{\mathrm{ph}}}\right)^{4} \exp \left(-\frac{E_{\mathrm{ph}}}{k_{\mathrm{B}} T}\right)
$$

where $I_{\mathrm{AS}}$ and $I_{\mathrm{S}}$ are the anti-Stokes and Stokes Raman intensities for the mode, $E_{0}$ and $E_{\mathrm{ph}}$ are the photon and phonon energies, respectively, and $A$ is a correction factor that accounts for the ratio of cross sections for Stokes and anti-Stokes Raman scattering.

At low incident laser power $(1 \mathrm{~mW})$, the temperature obtained, assuming $A=1$, was $289 \mathrm{~K}$, corresponding within experimental error to the ambient temperature of the water prior to laser illumination $(290 \mathrm{~K})$. We therefore assumed that the cross sections for Stokes and anti-Stokes scattering are similar and set $A=1$ for our subsequent analysis, which is reasonable since both Stokes and anti-Stokes cross sections are described by the same matrix element. Stokes and anti-Stokes spectra were obtained from the nanoparticle as incident laser power was increased, and the integrated areas of the $390 \mathrm{~cm}^{-1}$ mode peaks were converted to effective temperatures using eq 1 (Figure 2b). The temperature at the nanoparticle surface obtained in this manner was observed to increase smoothly and reversibly from 289 to $379 \mathrm{~K}( \pm 20 \mathrm{~K})$ with increasing laser power. This reversibility implies that, within this temperature range, the temperature of nanoparticle surface responds directly to the incident power, without any delay effects or hysteresis that would indicate a phase transition such as the formation of a vapor envelope around the nanoparticle, or a change in nanoparticle morphology or surface chemistry.

When the incident laser power was increased to $25 \mathrm{~mW}$, corresponding to the threshold for nanobubble formation inferred from the LSPR shift, the Raman spectra exhibited large changes in intensity. Both the Stokes and anti-Stokes intensities for the $390 \mathrm{~cm}^{-1}$ vibrational mode exhibit a dramatic jump after $60 \mathrm{~s}$ of illumination at this power level (Figure 2c). From the Stokes and anti-Stokes data and eq 1, we see that the observed spectral changes correspond to a rapid temperature increase from 400 to $465 \mathrm{~K}( \pm 3 \mathrm{~K})$ (in this regime of higher pump power levels, the error bars are within the symbols used to plot the data). This rapid temperature increase is consistent with the formation of a thermally insulating thin vapor layer around the nanoparticle. Once this initial layer is formed, the temperature of the nanoparticle and its surrounding vapor will increase until a new steady state determined by an energy balance between the incident power and the heat flow across the vapor/water interface of the vapor envelope, is reached. The final steady- state temperature of $465 \mathrm{~K}$, deduced from the Raman spectra, corresponds to the nanoparticle temperature, since the pMA is bound to the $\mathrm{Au}$ surface. Because this temperature is measured independently from the LSPR experiment and is significantly lower than the temperature onset of $647 \mathrm{~K}$ for spinodal decomposition of water, we do not believe that the measured LSPR shift is due to a local phase change of the water. The LSPR probing depth around a nanosphere of $100 \mathrm{~nm}$ only reaches a few tens of nanometers beyond the nanoparticle surface. Thus the measured LSPR shift cannot be explained by a large spinodal phase bubble with a weakly reduced refractive index but instead requires a substantial reduction of the refractive index in the immediate vicinity of the nanoparticle surface. Both of these observations support our conclusion that a vapor layer surrounds the nanoparticles.

A schematic of the system is shown in Figure 3a (for simplicity, the substrate is neglected). When the surface
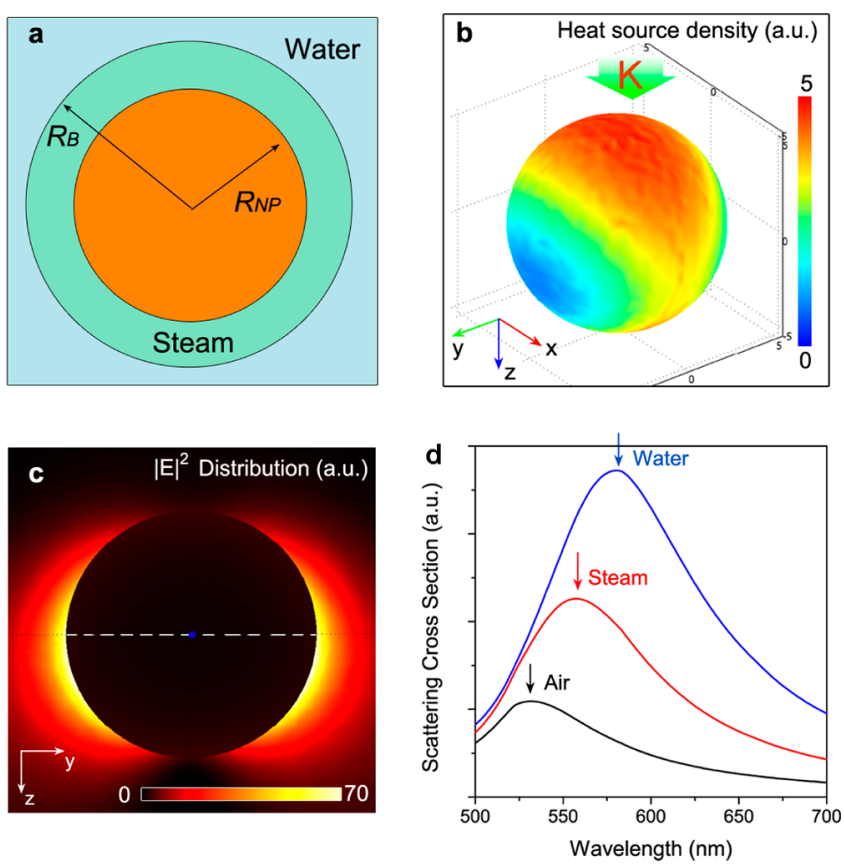

Figure 3. Nanobubble theoretical modeling. (a) Schematic of nanobubble formation around a nanoparticle under $532 \mathrm{~nm}$ resonant laser illumination. The nanobubble is a dielectric spacer layer of outer radius $R_{\mathrm{B}}$ between the nanoparticle surface (with radius $R_{\mathrm{NP}}$ ) and the surrounding water medium. (b) Simulated heat-source density for an illuminated $100 \mathrm{~nm}$ diameter $\mathrm{Au}$ nanoparticle immersed in water. Light is incident along the $z$ axis and linearly polarized along $y$. (c) Near-field intensity enhancement for a $100 \mathrm{~nm}$ diameter $\mathrm{Au}$ nanoparticle in water, relative to incident field intensity, under the same incidence and polarization conditions as in b. (d) Mie calculation of the scattering cross sections for a $100 \mathrm{~nm}$ diameter Au NP in air (black), surrounded by a steam bubble with outer radium $R_{\mathrm{B}}=60 \mathrm{~nm}$ (red), and in water (blue). The simulation for the steam layer is in good agreement with the experimental observations in Figure 1c.

plasmon of the nanoparticle is excited by a laser, a part of the incident energy dissipates elastically into scattered photons, while the remainder results in heat. The calculated heat source density, that is, the generated heating power per unit volume for a $100 \mathrm{~nm}$ diameter Au nanoparticle, immersed in water and illuminated by a linearly polarized plane wave with the incident $E$ field along the $y$ axis, is shown in Figure $3 \mathrm{~b}$. The "hottest" regions are at the "north" and "south" poles where the plasmon 
induced local currents are maximal. This is quite distinct from the regions of the nanoparticle surface with the largest plasmon-induced field enhancements, which occur on the sides of the sphere where the plasmon induced charge density is maximal (Figure 3c). Although the heat source density is not spherically uniform, the thermalization and heat diffusion in the gold particle are extremely fast relative to the heat diffusion through the steam and water, and thus we expect the metal nanoparticle to be effectively at a uniform temperature.

Since the observed LSPR blueshift of the nanoparticle is smaller than that for an infinite embedding medium of water vapor, we can use this information to estimate the bubble radius $R_{\mathrm{B}}$. Within an electrostatic dipole model, ${ }^{28,29}$ the redshift of the dipolar surface plasmon resonance is proportional to the relative permittivity change:

$$
\frac{\Delta \lambda_{\mathrm{B}}}{\Delta \lambda_{\mathrm{w}}}=\frac{\Delta \varepsilon_{\mathrm{B}}^{(\text {eff })}}{\Delta \varepsilon_{\mathrm{w}}} \equiv \frac{\varepsilon_{\mathrm{B}}^{(\text {eff })}-\varepsilon_{\mathrm{air}}}{\varepsilon_{\mathrm{w}}-\varepsilon_{\mathrm{air}}}
$$

where $\Delta \lambda_{\mathrm{B}}$ is the experimental redshift of the plasmon resonance for a nanoparticle in a finite nanobubble, $\Delta \lambda_{\mathrm{w}}$ is the redshift of the plasmon resonance in water relative to its value for steam, $\Delta \varepsilon_{\mathrm{B}}^{\text {(eff) }}$ is the permittivity increase for a finite nanobubble relative to steam, and $\Delta \varepsilon_{\mathrm{w}}$ is the permittivity increase for water relative to steam. The superscript "eff" denotes the effective permittivity change experienced by the nanosphere. Assuming the geometry depicted in Figure 3a, the values of $\varepsilon=1.0$ for steam (verified below), and $\varepsilon=1.77$ for water at room temperature, we performed Mie calculations ${ }^{30,31}$ to obtain theoretical scattering spectra for different values of the bubble radius. From comparison of the measured value of $\Delta \lambda_{\mathrm{B}}$ / $\Delta \lambda_{\mathrm{w}}=0.51$ (Figure 1c) to the calculated spectra, we deduce that the thickness of the vapor shell around a single $\mathrm{Au}$ nanoparticle is nominally $6.5 \mathrm{~nm}\left(R_{\mathrm{B}}=56.5 \mathrm{~nm}\right.$ ) (see Supporting Information, Figure S4). The LSPR measurement provides strong and consistent evidence that a nanosize bubble is generated. The spectral shift, calculated from Mie theory, agrees with the experimental scattering spectra using the concentric steam-water shell model where $R_{\mathrm{B}}=56.5 \mathrm{~nm}$ (Figure 3d).

The conventional model for plasmon induced bubble formation has recently been challenged. ${ }^{1}$ In the conventional model, the temperature increase at the particle surface is calculated as $\Delta T=\sigma I /\left(4 \pi k R_{\mathrm{NP}}\right)$, where $\sigma$ is the particle absorption cross section, $I$ is the incident intensity, and $k$ is the thermal conductivity of embedding material. ${ }^{26}$ By considering the presence of the glass substrate and using the thermal conductivity $\left(\sim 1.6 \mathrm{~W} \mathrm{~m}^{-1} \mathrm{~K}^{-1}\right)$ of glass around $500 \mathrm{~K}$, this model leads to an estimated particle temperature of around 774 $\mathrm{K}$. If the thermal conductivity of water is used, an even higher temperature results, in direct conflict with the observed $465 \mathrm{~K}$ from our Raman experiment. We notice that the calculation of temperature in this model is based on energy dissipation by a total conductive heat transfer process. However, it is wellknown that other heat transfer processes such as convection can also increase the effective thermal conductivity. Therefore we speculate that the discrepancy between our measured temperature and the result from the conventional model is due to the neglect of fluid convection. The conventional model assumes a stationary fluid and only conductive heat transfer.

Once the bubble is formed, there is an alternative approach for determination of the temperature by using the equation of state for saturated steam: Clausius-Clapeyron relation (CCR).
The CCR approach does not depend on the balance between the heating power and the dissipated power and thus is independent of the complexity of combinations of various heat transfer channels (through water, glass, convection, etc.). Instead, the CCR approach depends on only one unknown parameter: the bubble size which has been directly determined from our LSPR experiments. With the nanobubble radius determined, the temperature and pressure of the confined steam within the nanobubble can be estimated as follows. We assume that the system reaches steady state after bubble formation, as suggested in Figure $2 \mathrm{c}$ and $\mathrm{d}$. At equilibrium, the Laplace pressure due to the surface tension at the water-steam interface is balanced by the vapor pressure of the steam, which is a function of temperature given by the CCR. Hence, we have $C \exp \left(h / k_{\mathrm{B}} T_{\mathrm{s}}\right)=p_{0}+2 \gamma\left(T_{\mathrm{s}}\right) / R_{\mathrm{B}}$, where $C$ is a constant, $h$ is the enthalpy of water evaporation, $T_{s}$ is the temperature of saturated steam inside the bubble, $p_{0}$ is the atmospheric pressure $(1 \mathrm{~atm})$, and $\gamma$ is the surface tension coefficient (see Supporting Information, Text S1). The calculated steam temperature $T_{s}$ within the nanobubble is $464 \mathrm{~K}$, with a corresponding internal pressure of $1.49 \mathrm{MPa}(14.7 \mathrm{~atm})$. This large pressure is essentially a consequence of the large surface tension for a bubble of nanoscale dimensions. At this pressure and temperature, the dielectric permittivity of steam is close to 1.0 , consistent with the assumption made in the Mie calculation of steam layer thickness.

The temperature of the nanoparticle can also be calculated by applying Fourier's law at the gold-vapor interface: $P_{\mathrm{abs}}=$ $G_{\mathrm{g}, \mathrm{s}} S_{\mathrm{NP}}\left(T_{\mathrm{NP}}-T_{\mathrm{s}}\right)$, where $P_{\mathrm{abs}}$ is the power absorbed by the Au $\mathrm{NP}, G_{\mathrm{g}, \mathrm{s}}$ is the effective interfacial thermal conductance, $S_{\mathrm{NP}}$ is the surface area of the nanoparticle, and $T_{\mathrm{NP}}$ is the NP surface temperature. The temperature difference $\left(T_{\mathrm{NP}}-T_{\mathrm{s}}\right)$ is estimated to be approximately $72 \mathrm{~K}$ from a gold-steam interfacial thermal conductance of $275 \mathrm{MW} /\left(\mathrm{m}^{2} \mathrm{~K}\right.$ ) (see Supporting Information, Text S1, for a detailed calculation), resulting in an estimated nanoparticle surface temperature of $T_{\mathrm{NP}}=536 \mathrm{~K}$. The estimated average temperature of the molecular layer at the gold-vapor interface then was found to be $500 \mathrm{~K}$, which is a bit higher but close to the $465 \mathrm{~K}$ obtained from our SERS measurements. This difference is most likely due to our neglect of the dielectric substrate, which serves as a heat sink as compared with water. It is worth noting that the analysis of the interfacial thermal conductance (Supporting Information) is an essential step for final determination of the surface temperature of the gold nanosphere from the steam temperature calculated in our approach. In fact, there are two competing cooling rates: one controlled by the effusivity of the fluid and one determined by the interface effect as discussed by Ge et al. ${ }^{32}$ Such a competition is very important for transient temporal evolution of temperature, i.e., in a pulsed-laser study. ${ }^{32}$ However, it is not relevant here because we use CW laser illumination, and the system is in steady state after the bubble is formed. In steady state, the heating power is balanced by the power dissipated through fluid effusivity and heat transfer across the interfaces. There is no need to consider the competition between these two mechanisms since in a steady state we are not interested in the cooling rates but the temperature difference across the interface.

To study the transition from discrete nanobubbles localized on their individual, parent nanoparticles to optically observable, micrometer sized bubbles, the incident light intensity needs to be increased above the $25 \mathrm{~mW}$ threshold and/or the nanoparticle density on the substrate needs to be increased. 
A time series of images under these new conditions, resulting in the formation of a microbubble, is shown in Figure 4a. After
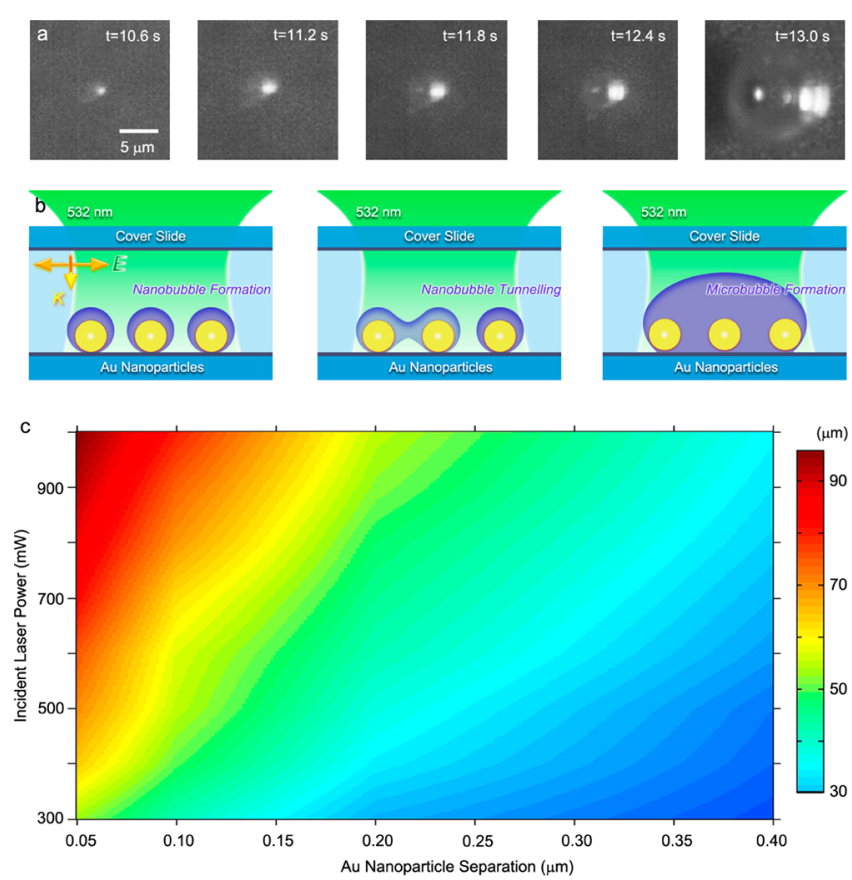

Figure 4. Coalescence of nanobubbles into micrometer-sized bubbles. (a) Time series of microbubble generation. The nanoparticle areal density is $3 \times 10^{8} \mathrm{~cm}^{-2}$ (average NP-NP separation $\sim 0.6 \mu \mathrm{m}$ ). (b) Schematics depicting microbubble formation due to the coalescence of neighboring nanobubbles produced on individual $\mathrm{Au}$ nanoparticles. (c) Experimental diagram of the dependence of final bubble size and formation time on incident laser power and average nanoparticle separation, where the color indicates final bubble diameter (scale bar on right). The $532 \mathrm{~nm}$ light was focused on a $5 \mu \mathrm{m}$ diameter spot.

illumination ( $t=10.6 \mathrm{~s})$, a $1 \mu \mathrm{m}$ bright spot (bubble) appears in the image, and after $t=13.0 \mathrm{~s}$ it has expanded to a microbubble of $10 \mu \mathrm{m}$ diameter, with a volume encompassing approximately 5000 nanoparticles. We believe that the origin of the observed microbubbles is the coalescence of individual nanobubbles centered on adjacent nanoparticles, as illustrated in Figure $4 \mathrm{~b}$. With increasing light intensity, the radii of the nanobubbles increase. If the interparticle spacing is sufficiently small, nanobubbles centered on adjacent nanoparticles will touch and coalesce, resulting in the formation of a larger microbubble encompassing several nanoparticles. Similar attraction-and-coalescence has also been observed for microbubbles.

The final size of the microbubble is a function of both incident power and nanoparticle separation. This is shown in the contour plot in Figure 4c, where the color map represents final bubble size as a function of these two parameters (the incident laser spot size is $5 \mu \mathrm{m}$ ). This contour plot is a result of an extended series of experimental measurements for multiple interparticle distances and input laser powers (Supporting Information, Table 1). Generally speaking, higher incident powers $(\geq 400 \mathrm{~mW})$ and shorter interparticle separations $(\leq 0.4$ $\mu \mathrm{m})$ result in a larger bubble size. We observe a strong sensitivity to interparticle distance: for large interparticle separations, only small microbubbles can be observed, even at the highest laser powers. This reflects the fact that the total energy absorbed within the laser spot of $5 \mu \mathrm{m}$ is due to the total number of nanoparticles within the beam spot, a number which grows as the inverse square of the interparticle separation. In an isotropic environment we would anticipate that, for a specific bubble radius (single-color contours of Figure $4 \mathrm{c}$ ), the input power would scale with the inverse square of the interparticle distance. The data measured here deviates from that simple scaling, most likely due to the additional loss introduced by the nanoparticle substrate.

In conclusion, we have examined the phenomenon of lightinduced vapor generation at a plasmonic nanoparticle surface, from the initial formation of a nanoscale vapor envelope around an individual nanoparticle to the formation of micrometer-scale bubbles due to coalescence of the expanded vapor envelopes. Our single-particle studies of vapor generation enable a detailed characterization of the size, pressure, and temperature of the nascent nanobubble formed at a nanoparticle surface. The combination of quantitative measurements at the single nanoparticle level and rigorous analysis provides us with the first detailed picture of the vapor generation process at its initial stages. Our approaches for characterizing vapor formation should prove to be useful in the study of other local energetic processes, such as catalysis and local chemical or materialgrowth reactions.

Methods. Sample Preparation. Au nanoparticles with differing diameters ranging from 30 to $200 \mathrm{~nm}$ (NanoXact, Ted Pella, Inc.) were randomly dispersed and immobilized on the microchamber bottom surface, which was functionalized with poly(4-vinyl pyridine)(PVP). For single nanoparticle detection, a transmission electron microscope (TEM) sample grid was used to fabricate fiduciary marks by e-beam evaporation. To prevent the influence from other $\mathrm{Au}$ nanoparticles, the selected nanoparticle was the only one found within the $10 \mu \mathrm{m} \times 10 \mu \mathrm{m}$ sampling area. The microchamber was fabricated using $3 \mathrm{M}$ tape with a diameter of $1 \mathrm{~cm}$ and thickness of $80 \mu \mathrm{m}$. The chamber was sealed by a thin glass cover slide. The temperature of the microchamber and of the injected water was kept constant at $285 \mathrm{~K}$. A thermograph was used to monitor any temperature change during the experimental measurements.

Experimental Setup and Measurements. The sample was placed on a piezoelectric positioning stage (Nanonics Co.) held by a $x y z$ coarse adjustment stage (Newport Co.). A long working distance $(12 \mathrm{~mm})$ objective lens (Mitutoyo NIR $100 \mathrm{X}$ ) with a numerical aperture 0.5 was used to focus a 532 $\mathrm{CW}$ laser beam onto the Au NP at normal incidence. The focus diameter was measured by moving a razor edge with the piezoelectric positioning stage, assuming a Gaussian beam profile. A white light source (MI-150, Edmond Optics) was used to illuminate the sample at oblique incidence. The darkfield scattering signals passed through the same objective lens and were detected by a monochromator (Acton SP2150, PI) with a CCD camera (PIXIS400, PI). For the Raman measurements, a monolayer of $p$-mercaptoaniline (pMA) $(\sim 0.3 \mathrm{~nm})$ was bound to the $\mathrm{Au}$ nanoparticle surface, and SERS measurements were performed with a microchamber filled with pure water. The signal was recorded by a Raman spectrometer (Acton SpectraPro2300i, PI) along the same experimental optical path. Microbubble generation was imaged using a CCD camera. 


\section{ASSOCIATED CONTENT}

\section{S Supporting Information}

Figure S1: The surface plasmon resonance spectrum of a 100 $\mathrm{nm}$ nanoparticle in liquid water at 20 and $100{ }^{\circ} \mathrm{C}$; Figure S2: Calculated Mie absorption efficiency for different size $\mathrm{Au}$ nanoparticles; Figure S3: Raman spectra for Figure 2a and $\mathrm{c}$ in the main text; Figure S4: Calculation of nanobubble thickness; Text S1: Calculation of temperature inside a nanobubble; Table S1: Experimental data for Figure $4 \mathrm{c}$ in main text; Movie S1: Microbubble coalescence. This material is available free of charge via the Internet at http://pubs.acs.org.

\section{AUTHOR INFORMATION}

\section{Corresponding Author}

*E-mail: zhyfang@rice.edu; nordland@rice.edu; halas@rice. edu.

\section{Author Contributions}

Z.F. and Y.R.-Z. contributed equally.

\section{Notes}

The authors declare no competing financial interest.

\section{ACKNOWLEDGMENTS}

We thank the following agencies for their support of this research: The Robert A. Welch Foundation (C-1220, NH) and (C-1222, PN), The Air Force Office of Scientific Research (FA9550-10-1-0469, NH), the DoD NSSEFF (N00244-09-10067, NH and PN), the NIH NCI (U01 CA151886-01, NH and $\mathrm{PN}$ ), and the Bill and Melinda Gates Foundation (OPP1044571, NH). Work at AMOLF is part of the research program of FOM and is also supported by the European Research Council.

\section{REFERENCES}

(1) Neumann, O.; Urban, A. S.; Day, J.; Lal, S.; Nordlander, P.; Halas, N. J. Solar Vapor Generation Enabled by Nanoparticles. ACS Nano 2013, 7, 42-49.

(2) Polman, A. Solar Steam Nanobubbles. ACS Nano 2013, 7, 1518

(3) Govorov, A. O.; Richardson, H. H. Generating heat with metal nanoparticles. Nano Today 2007, 2, 30-38.

(4) Adleman, J. R.; Boyd, D. A.; Goodwin, D. G.; Psaltis, D. Heterogenous Catalysis Mediated by Plasmon Heating. Nano Lett. 2009, 9, 4417-4423.

(5) Boyd, D. A.; Greengard, L.; Brongersma, M.; El-Naggar, M. Y.; Goodwin, D. G. Plasmon-assisted chemical vapor deposition. Nano Lett. 2006, 6, 2592-2597.

(6) Baffou, G.; Quidant, R.; Garcia de Abajo, F. J. Nanoscale Control of Optical Heating in Complex Plasmonic Systems. ACS Nano 2010, 4, 709-716.

(7) Boyd, D. A.; Adleman, J. R.; Goodwin, D. G.; Psaltis, D. Chemical separations by bubble-assisted interphase mass-transfer. Anal. Chem. 2008, 80, 2452-2456.

(8) Christopher, P.; Xin, H. L.; Linic, S. Visible-light-enhanced catalytic oxidation reactions on plasmonic silver nanostructures. Nat. Chem. 2011, 3, 467-472.

(9) Huth, F.; Schnell, M.; Wittborn, J.; Ocelic, N.; Hillenbrand, R. Infrared-spectroscopic nanoimaging with a thermal source. Nat. Mater. 2011, 10, 352-356.

(10) Ibrahimkutty, S.; Kim, J.; Cammarata, M.; Ewald, F.; Choi, J.; Ihee, H.; Plech, A. Ultrafast Structural Dynamics of the Photocleavage of Protein Hybrid Nanoparticles. ACS Nano 2011, 5, 3788-3794.

(11) Jackson, J. B.; Halas, N. J. Surface-enhanced Raman scattering on tunable plasmonic nanoparticle substrates. Proc. Natl. Acad. Sci. U.S.A. 2004, 101, 17930-17935.
(12) Lee, J.; Govorov, A. O.; Kotov, N. A. Nanoparticle assemblies with molecular springs: A nanoscale thermometer. Angew. Chem., Int. Ed. 2005, 44, 7439-7442.

(13) Li, C.; Wang, Z.; Wang, P. I.; Peles, Y.; Koratkar, N.; Peterson, G. P. Nanostructured copper interfaces for enhanced boiling. Small 2008, 4, 1084-1088.

(14) Lim, Z. Z. J.; Li, J. E. J.; Ng, C. T.; Yung, L. Y. L.; Bay, B. H. Gold nanoparticles in cancer therapy. Acta Pharmacol. Sin. 2011, 32, 983-990.

(15) Oron-Carl, M.; Krupke, R. Raman spectroscopic evidence for hot-phonon generation in electrically biased carbon nanotubes. Phys. Rev. Lett. 2008, 100, 127401.

(16) Richardson, H. H.; Carlson, M. T.; Tandler, P. J.; Hernandez, P.; Govorov, A. O. Experimental and Theoretical Studies of Light-toHeat Conversion and Collective Heating Effects in Metal Nanoparticle Solutions. Nano Lett. 2009, 9, 1139-1146.

(17) von Maltzahn, G.; Park, J.-H.; Lin, K. Y.; Singh, N.; Schwöppe, C.; Mesters, R.; Berdel, W. E.; Ruoslahti, E.; Sailor, M. J.; Bhatia, S. N. Nanoparticles that communicate in vivo to amplify tumour targeting. Nat. Mater. 2011, 10, 545-552.

(18) Ward, D. R.; Corley, D. A.; Tour, J. M.; Natelson, D. Vibrational and electronic heating in nanoscale junctions. Nat. Nanotechnol. 2011 $6,33-38$

(19) Atwater, H. A.; Polman, A. Plasmonics for improved photovoltaic devices. Nat. Mater. 2010, 9, 205-213.

(20) Erickson, D.; Sinton, D.; Psaltis, D. Optofluidics for energy applications. Nat. Photonics 2011, 5, 8.

(21) Schuller, J. A.; Taubner, T.; Brongersma, M. L. Optical antenna thermal emitters. Nat. Photonics 2009, 3, 658-661.

(22) Carlson, M. T.; Green, A. J.; Richardson, H. H. Superheating Water by CW Excitation of Gold Nanodots. Nano Lett. 2012, 12, 1534-1537.

(23) Lukianova-Hleb, E.; Hu, Y.; Latterini, L.; Tarpani, L.; Lee, S.; Drezek, R. A.; Hafner, J. H.; Lapotko, D. O. Plasmonic Nanobubbles as Transient Vapor Nanobubbles Generated around Plasmonic Nanoparticles. ACS Nano 2010, 4, 2109-2123.

(24) Anderson, L. J. E.; Mayer, K. M.; Fraleigh, R. D.; Yang, Y.; Lee, S.; Hafner, J. H. Quantitative Measurements of Individual Gold Nanoparticle Scattering Cross Sections. J. Phys. Chem. C 2010, 114, $11127-11132$.

(25) Sonnichsen, C.; Geier, S.; Hecker, N. E.; von Plessen, G.; Feldmann, J.; Ditlbacher, H.; Lamprecht, B.; Krenn, J. R.; Aussenegg, F. R.; Chan, V. Z.-H.; Spatz, J. P.; Möller, M. Spectroscopy of single metallic nanoparticles using total internal reflection microscopy. Appl. Phys. Lett. 2000, 77, 2949-2951.

(26) Govorov, A. O.; Zhang, W.; Skeini, T.; Richardson, H.; Lee, J.; Kotov, N. A. Gold nanoparticle ensembles as heaters and actuators: melting and collective plasmon resonances. Nanoscale Res. Lett. 2006, $1,84-90$.

(27) Galloway, C. M.; Le Ru, E. C.; Etchegoin, P. G. Single-molecule vibrational pumping in SERS. Phys. Chem. Chem. Phys. 2009, 11, $7372-7380$.

(28) Jain, P. K.; El-Sayed, M. A. Noble Metal Nanoparticle Pairs: Effect of Medium for Enhanced Nanosensing. Nano Lett. 2008, 8, 4347-4352.

(29) Miller, M. M.; Lazarides, A. A. Sensitivity of metal nanoparticle surface plasmon resonance to the dielectric environment. J. Phys. Chem. B 2005, 109, 21556-21565.

(30) Garcia de Abajo, F. J. Multiple scattering of radiation in clusters of dielectrics. Phys. Rev. B 1999, 60, 6086-6102.

(31) Myroshnychenko, V.; Rodríguez-Fernández, J.; Pastoriza-Santos, P.; Funston, A. M.; Novo, C.; Mulvaney, P.; Liz-Marzán, L. M.; García de Abajo, F. J. Modelling the optical response of gold nanoparticles. Chem. Soc. Rev. 2008, 37, 1792-1805.

(32) Ge, Z. B.; Cahill, D. G.; Braun, P. V. AuPd metal nanoparticles as probes of nanoscale thermal transport in aqueous solution. J. Phys. Chem. B 2004, 108, 18870-18875. 